\title{
Identificación de Cangahuas para su recuperación mediante estudio multicriterio y constatación in situ en comunas del volcán Ilaló
}

\author{
Iván Palacios-Orejuela ${ }^{\mathrm{a}}$, Dennis Ushiña-Huera ${ }^{\mathrm{a}}$, David Carrera-Villacrés ${ }^{\mathrm{a}, \mathrm{b}}$ \\ ${ }^{a}$ Departamento de Ciencias de la Tierra y la Construcción, Carrera de Ingeniería Geográfica y del Medio Ambiente, \\ CIGMA, Grupo de Investigación en Contaminación Ambiental (GICA), Universidad de las Fuerzas Armadas ESPE, \\ Sangolquí, Ecuador \\ ${ }^{\text {b }}$ Facultad de Ingeniería Geológica, Minas, Petróleo y Ambiental. FIGEMPA, Carrera de Ingeniería Ambiental, Universidad \\ Central del Ecuador, Quito, Ecuador \\ ifpalacios@espe.edu.ec, dpushiña@espe.edu.ec, dvcarrera@espe.edu.ec
}

\begin{abstract}
Resumen-En la Sierra del Ecuador se ha agravado el problema de erosión del suelo como consecuencia de malas prácticas agrícolas, presencia de fuertes pendientes y su propia composición. Debido al origen volcánico de los suelos andinos es común encontrar cangahuas, tobas endurecidas que al aflorar ocasionan problemas a la agricultura. La falta de nuevas tierras productivas, en conjunto con la expansión agrícola, generan la necesidad de rehabilitar las áreas afectadas para mejorar la calidad de vida de los habitantes y recuperar el paisaje. Un claro ejemplo son las laderas del Volcán Ilaló, cantón Quito, donde la cobertura vegetal y tierras productivas han venido en decremento en las últimas décadas, así nace el proyecto de recuperación de cangahuas para evitar el deterioro del terreno, este consiste en la roturación del suelo para permitir la oxigenación e ingreso de nutrientes y agua, convirtiéndolo en tierra productiva. El objetivo del presente estudio fue encontrar áreas adecuadas para su recuperación y creación de huertos comunales, en las comunas de Angamarca, Alangasí, San Francisco de Baños y La Toglla localizadas a las faldas del volcán Ilaló, mediante la aplicación del Análisis Multicriterio específicamente el método del Proceso Analítico Jerárquico de Thomas Saaty. Para esto fueron identificadas cinco variables geográficas con mayor relevancia: pendiente, hidrografía, vialidad, población y forma del relieve. En las parcelas resultantes se aplicó muestreo aleatorio simple para a través de la construcción de calicatas y perfiles, verificar la presencia de cangahua con espesor de al menos $60 \mathrm{~cm}$. La existencia de parcelas consideradas como óptimas por cumplir las condiciones establecidas para la remediación fue constatada en tres de las comunas, con una profundidad promedio de cangahua mayor al metro y un área total para implementar la remediación de $\mathbf{2 0 . 8 6}$ ha. Finalmente, se propuso este trabajo como base para posteriores proyectos.
\end{abstract}

Palabras Claves-Análisis multicriterio, cangahua, comunas, muestreo, recuperación.

Abstract-The problem of soil erosion has worsened as a result of bad agricultural practices, the presence of steep slopes and its own composition in the highlands of Ecuador. Cangahuas are common to find due to the volcanic origin of Andean soils. When they appear cause serious problems to agriculture production. Because of lack of new productive lands, together with the agricultural expansion, restore affected areas by cangahua formation and soil erosion has become a priority. Recovering soils production is needed to improve the quality of life of the inhabitants and recover the landscape. An example of this is the slopes of the Ilaló Volcano in Quito, where the vegetation and productive land have been decreasing in recent decades. Cangahuas recovery project began in 2017 to prevent land worsening and it involves clearing the soil to allow oxygenation and nutrients and water go into soils converting it into productive land. The objective of this study was to find suitable areas to recovery cangahua soils degradation and creation of community orchards, in the communes of Angamarca, Alangasí, San Francisco de Baños and La Toglla. These communities are located at the foot of the Ilaló volcano. Recovery areas were selected by application of Multicriteria Analysis, specifically the Analytical Hierarchy Process method by Thomas Saaty. Five geographic variables were identified: slope, hydrography, roads, population and topography. A simple random sampling was applied to pits and soil profiles to verify cangahua soils with a thickness of at least $60 \mathrm{~cm}$. The optimal land parcels that met soils conditions to remediation was found in three of the communities. These communities presented an average over a meter of depth in cangahua. Total area to implement the remediation was $\mathbf{2 0 . 8 6}$ ha. Finally, this work was proposed as a basis for subsequent projects.

Keywords-Cangahua, communities, multicriteria analysis, recovery, sampling.

\section{INTRODUCCIÓN}

Mantener los suelos aptos para la agricultura y reducir la erosión son desafíos que afronta actualmente la sierra centro del Ecuador, donde se buscan medios y tecnologías para el máximo aprovechamiento del espacio. Precisamente en esta región, su pasado volcánico ha originado formaciones edafológicas con características físicas y químicas que impiden su labranza. La cangahua, vocablo quichua que significa “tierra dura estéril”, es una formación geológica derivada de los piroclastos del Cuaternario, que se encuentra en la superficie del suelo con un alto grado de endurecimiento [1]. Se las define como suelos volcánicos endurecidos ubicados en la parte septentrional del callejón interandino formados por piroclastos [2]. Vera y López (1992), la describen como una roca blanda y porosa resultante de la diagénesis parcial del material volcánico explosivo fino procedente de flujos piroclásticos la cual es originada por caídas piroclásticas, con tamaño de ceniza $<2 \mathrm{~mm}$ [3]; definiciones que coinciden con dos grandes naturistas del Ecuador, Woff y Sauer, quienes consideraban a la cangahua como una toba endurecida. Por lo general, las cangahuas se encuentran en los flancos medios y bajos de volcanes, éstas, son capas volcánicas parcialmente alteradas y compactadas tras procesos geológicos y edafológicos, su grosor varía desde los centímetros hasta los metros, de consistencia dura por presencia de carbonatos, silicio, 
aluminio y hierro, y falta de precipitación [4]. La penetración del agua es mínima, una simulación demostró que el escurrimiento puede alcanzar hasta un $80 \%$ cuando la intensidad de lluvia es de $20 \mathrm{~mm} / \mathrm{h}$, lo que la hace susceptible a la erosión hídrica [1]. Desde el punto de vista químico, las cangahuas poseen muy bajo contenido de nitrógeno y materia orgánica (MO) [5], además, los suelos derivados de la cangahua tienen un $\mathrm{pH}$ neutro y contienen calcio $(9.5-14.4 \mathrm{meq} / 100 \mathrm{~g})$ y potasio de intercambio (0.81 - $1.94 \mathrm{meq} / 100 \mathrm{~g}$ ) [1].

A partir del crecimiento poblacional los agricultores se han visto en la necesidad de expandirse sobre este tipo de subsuelo, es así como un 15\% de formaciones de cangahua en el Ecuador son usadas para actividad agrícola [6]. Varios estudios han abordado esta temática, enfocados principalmente en la producción agrícola sobre estos sustratos [7], [8], entre otros. Pese a esto, la información relacionada a las cangahuas en el Ecuador aún es escasa. Cifras obtenidas en estudios anteriores muestran la existencia de aproximadamente 240000 ha., de las cuales 36000 ha., están en la superficie del suelo gracias a la erosión sufrida, y 44000 ha., estarían cubiertas por casi 20 cm de suelo apto para cultivar [9]. En base a lo expuesto, se dice que las cangahuas son un subsuelo infértil y constituyen una problemática para el desarrollo agrícola del sector rural, sin embargo, a través de trabajo manual o con maquinaria es posible cultivar sobre estos con escasos insumos agregados [9]. La recuperación de cangahua implica establecer prácticas de roturación (labranza), que permitan oxigenar la tierra y mejorar la capacidad de suministro de nutrientes al suelo, ya sea por acción humana o natural con sus posteriores labores de conservación para mantener el sustrato [10].

Por consiguiente, el objetivo del presente trabajo fue identificar las parcelas con cangahuas adecuadas para su recuperación y posterior creación de huertas comunales, mediante un estudio multicriterio y constatación in situ en comunas del volcán Ilaló, específicamente los asentamientos de Angamarca, Alangasí, San Francisco de Baños y La Toglla.

\section{MÉTODO}

\section{A. Descripción del área de estudio}

La zona de estudio correspondió a las parroquias rurales de Alangasí, Guangopolo y La Merced, pertenecientes al cantón Quito, provincia de Pichincha. Dentro de las cuales están localizadas las comunas de Angamarca, Alangasí, San Francisco de Baños y La Toglla, que comprenden una superficie de $29 \mathrm{~km}^{2}$ [11].

\section{B. Análisis Multicriterio}

Es una herramienta que permite comparar múltiples variables entre ellas para facilitar la toma de decisiones sobre un objetivo planteado [12]. Dentro del Análisis Multicriterio se encuentra el método denominado Proceso Analítico Jerárquico, AHP (Analytical Hierarchy Process) dado por Thomas Saaty, el cual realiza una comparación por pares de los criterios a partir de una matriz nxn [13]. Posterior a esta comparación, se establecen los pesos, los cuales proporcionan una medida cuantitativa de los juicios de valor.

Se trabajó con cinco variables principales para la determinación de las zonas óptimas. La variable "Pendiente" debe ser menor al 25\% para permitir el ingreso de maquinaria pesada; la facilidad de acceso a las parcelas está dada por la cercanía a la variable "Vías". La variable "Población área” se tomó en cuenta para no interferir en zonas pobladas, mientras con "Forma del relieve" se estableció la presencia o ausencia de zonas protegidas, agrícolas o semejantes, y con "Ríos y drenajes" se verificó que no existieran estos accidentes geográficos dentro de la zona de interés. La información base se obtuvo a partir de datos en formato .shp a escala 1:25000 y un modelo digital de elevación (DEM) a escala 1:5000.

Una vez establecidas las variables, se ponderó cada una de las mismas, las que, con mayor influencia al momento de tomar la decisión fueron puntuadas con valores más altos. Para ello se utilizó la matriz de Saaty, cuyos valores se encuentra en un rango de 1 a 9, donde 1 es "igual importancia” y 9 es “extremadamente importante” [14]; se puede delimitar los valores máximos y mínimos en función del número de variables, por lo cual se tomó valores entre 1 y 5 , con el mismo significado.

Para dar el peso real en función de la importancia de las variables a relacionar, se utilizó el software IDRISI 17.0, al interior del cual fue ingresada la matriz en la herramienta WEIGHT - AHP. Los pesos resultantes al aplicar la metodología de Saaty se observan en la Fig. 1:

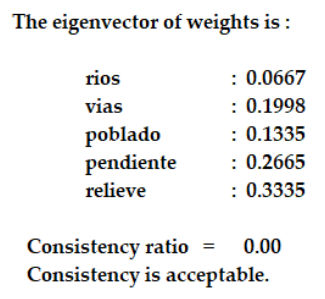

Fig. 1. Pesos de las variables mediante Saaty con IDRISI 17.0

Una vez calculados los pesos se procedió a hallar las zonas adecuadas para la recuperación de cangahua con ayuda del software ArcGis 10.2. Las capas que contenían a las variables "Ríos y drenajes” y "Vías" se añadió un área de influencia mediante buffers de 20 y 100 metros respectivamente. La capa de "Pendientes" fue calculada a partir del DEM de la zona de estudio y se tomó únicamente las pendientes con una inclinación de hasta 25\%. En tanto, a las capas de "Poblados área" y "Forma de relieve" no se aplicó ningún buffer. Los archivos fueron transformados de formato vector (.shp) a ráster (.tif) para reclasificar los pixeles en valores de 0 y 1 .

Mediante álgebra de mapas de las coberturas reclasificadas se obtuvieron las parcelas que cumplen con las condiciones dadas. A manera de ejemplo se presentan los polígonos obtenidos de la Comuna de Alangasí en la Fig. 2. 


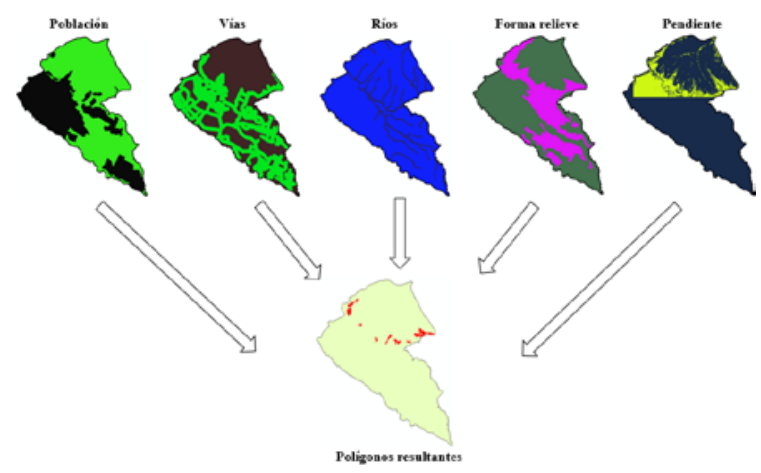

Fig. 2. Algebra de mapas para la obtención de polígonos finales

\section{Muestreo del suelo}

Debido a la escasa información acerca de la población, que, para casos de este estudio fue el desconocimiento de la distribución de la cangahua en el terreno, se eligió un muestreo aleatorio simple, mismo que basado en estadística de probabilidad permite estudiar poblaciones relativamente pequeñas y homogéneas [15].

Antes del muestreo, se realizó una visita de campo exploratoria de las parcelas determinadas en la sección II-B, con la ayuda de los comuneros para seleccionar aquellas parcelas localizadas dentro de los límites de las comunas (límites no muy claros) y que presenten a su vez, las mejores condiciones para el trabajo en cada comuna. Como ejemplo, en la Alangasí, de las 16 parcelas obtenidas, únicamente se ubicaron 3 parcelas útiles, las cuales se pueden observar en la Fig. 3:

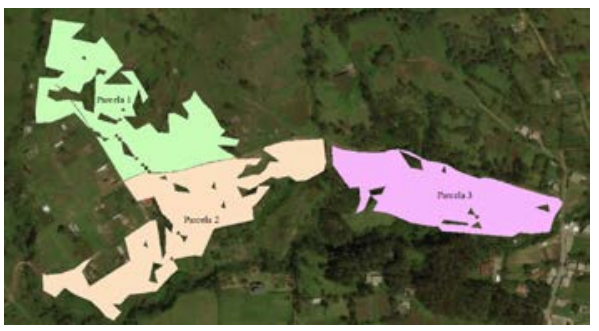

Fig. 3. Parcelas dentro de la Comuna de Alangasí

A través de un GPS navegador Garmin Etrex 20, se tomó las coordenadas de los límites de los terrenos seleccionados al interior de las parcelas, en los cuales se trazó una grilla de 3x3 metros sobre el mismo. Según lo establecido en la Norma ecuatoriana de calidad ambiental del recurso suelo y criterios de remediación para suelos contaminados, se hizo un muestreo aleatorio en el que se escogió un cuadrante para aplicar nuevamente un muestreo aleatorio simple con polígonos de $1 \mathrm{x} 1 \mathrm{~m}^{2}$. Dentro del cuadrante seleccionado y en base al TULSMA, se cavó una calicata con una profundidad mínima de $60 \mathrm{~cm}$, para observar los horizontes y la profundidad a la que se encontraba la cangahua desde la superficie.

Además, se realizaron varios perfiles, con ayuda de cunetas y zanjas ya existentes en la zona, que tenían alturas de hasta $3 \mathrm{~m}$. Se procedió a limpiar y cavar la pared de los perfiles para observar el material sin alteración y realizar el mismo análisis de profundidad y cambio de horizonte en el suelo, efectuado en las calicatas. Finalmente, se recolectó muestras de suelos de todos los puntos escogidos para el estudio, con un total de 5 en cada comuna, de acuerdo con el TULSMA, libro VI y Anexo 2.

\section{ANÁLISIS DE RESULTADOS}

El análisis multicriterio determinó para la comuna de Alangasí un total de 3 polígonos óptimos para su intervención, en los que se realizó 2 calicatas $(70 \mathrm{~cm}$ de profundidad) y 3 perfiles con una profundidad de $2.10 \mathrm{~m}$, donde el horizonte visible fue en su mayoría cangahua de color café claro amarillento y textura arcillosa. Para efectos de este trabajo, era necesaria una profundidad mínima de 60 cm., que permita el uso de maquinaria pesada.

En promedio la profundidad de la cangahua fue de 1.12 m, además, se consideró un posible cuarto lote en el cual se visualizó la presencia de cangahua y se propuso como opción adicional. En la Tabla I se presentan los polígonos resultantes para esta comuna. El área total que se obtuvo de terreno apto para la recuperación fue de 1.02 ha., y si se considera la parcela opcional, el área de trabajo aumenta a 1.58 ha.

TABLA I

ÁREA DE LAS PARCELAS

\begin{tabular}{ccc}
\hline \hline Parcela & Propietario & Área $\left(\mathbf{m}^{\mathbf{2}}\right)$ \\
\hline 1 & Comuna & 2931.68 \\
2 & Comunero & 4183.91 \\
3 & Comunero & 5566.61 \\
4 & Comunero & 3133.02 \\
\hline \hline
\end{tabular}

Para las tres comunas restantes se determinaron 4 parcelas en cada una. Las áreas totales y el espesor promedio de la cangahua (EPC) para Angamarca y San Francisco de Baños se muestran en la Tabla II. Los ocho polígonos de estas comunas se ubican en terrenos de propiedad comunal con cangahuas aflorante y subaflorante, de iguales características físicas a las de la comuna de Alangasí.

TABLA II

RESUlTADOS OBTENIDOS EN LAS COMUNAS

\begin{tabular}{ccc}
\hline \hline & Angamarca & San Francisco de Baños \\
\hline Calicatas & 3 & 5 \\
Perfiles & 2 & 0 \\
EPC (m) & 1 & 1.2 \\
Área total (ha) & 16.24 & 3.05 \\
\hline \hline
\end{tabular}

En La Toglla, no se halló cangahua cerca de la superficie en ninguno de los cuatro polígonos, mediante las 2 calicatas y 3 perfiles realizados, se determinó como horizonte predominante al A, es decir suelo con contenido de $\mathrm{MO}$, cuyo espesor promedio fue de $1 \mathrm{~m}$. Sin embargo, se constató el deterioro de los mismos por las condiciones adversas que afectan a toda el área de las laderas del volcán y propensos a la erosión. El área total de los cuatro polígonos en La Toglla fue de 3.63 ha.

De acuerdo al trabajo de campo realizado, se determinó que la tipología de cangahua predominante en el estudio, según la clasificación dada por Vera y López (1992), correspondió al tipo primario de flujo de ceniza, debido a su color café amarillento, presencia de MO (>1\%), bimodal de arena a limo, con presencia de líticos de pómez [3], lo que coincide con trabajos anteriores [16].

Finalmente, en las comunas estudiadas fueron construidas 12 calicatas y 8 perfiles, en los cuales se verificó la presencia de cangahua para las comunas de Alangasí, Angamarca y San Francisco de Baños con una extensión de 
20.86 ha., donde Angamarca fue la comuna con la mayor área (16.23 ha.).

\section{CONCLUSIONES}

A través del estudio multicriterio realizado en base a cinco variables y al muestreo aplicado se definieron al menos cuatro parcelas óptimas para la recuperación de cangahuas y la creación de huertos de uso comunal, dentro de las comunas de Alangasí, Angamarca y San Francisco de Baños. En la comuna La Toglla, se determinó que las parcelas aún poseen un adecuado horizonte agrícola, por lo que no es necesaria una roturación, pero si es indispensable el implementar prácticas agrícolas que mejoren sus características y protejan el recurso suelo de la erosión. La cangahua en su mayoría se encuentra aflorante con una profundidad promedio de $1.12 \mathrm{~m}$, lo que permitirá llevar a cabo la roturación del suelo con uso de maquinaria sin problemas de encontrarse con roca madre. Pese a la presencia de cangahuas en gran parte de las comunas, las principales limitaciones son las pendientes pronunciadas y el difícil acceso, para estas áreas es posible realizar una recuperación sin el uso de maquinaria pesada. Finalmente, se propuso este trabajo como base para posteriores proyectos.

\section{REFERENCIAS}

[1] E. Custode, G. De Noni, G. Trujillo y M. Viennot, "La cangahua en el Ecuador: caracterización morfo-edafologica y comportamiento frente a la erosion The cangahua in Ecuador: morpho-edaphologic characterization and susceptibility to erosion," Terra, vol. 10, pp. 332-346, 1992.

[2] C. Zebrowski, "Los suelos con cangahua en el Ecuador.” Memorias del III Simposio Internacional sobre Suelos volcánicos endurecidos, pp. 128-137, 1997.

[3] R. Vera y R. López, “Tipología de la cangahua,” Terra, vol. 10, pp. 113-119, 1992.

[4] J. Hidrobo, M. Da Costa, C. Prat, G. Trujillo, J. Moreno y C. Ortega, "Sistemas de producción en áreas con cangahua habilitada en la Sierra Norte del Ecuador,” Siembra, vol. 2, pp. 116-127, 2015.

[5] C. Prat, R. Duponnois y S. Caquineau, "CANGAHUAS Recuperación agrícola participativa de suelos erosionados de la sierra ecuatoriana,” 2013. [En línea]. Available: http://www.ecuador.ird.fr/actividades/proyectos-deinvestigacion/agricultura/cangahuas.-recuperacion-agricolaparticipativa-de-suelos-erosionados-de-la-sierra-ecuatoriana.

[6] P. Podwojewski y N. Germain, "Short-term effects of management on the soil structure in a deep tilled hardened volcanic-ash soil (cangahua) in Ecuador," European Journal of Soil Science, vol. 56, pp. 39-51, 2004. DOI: 10.1111/j.1365-2389.2004.00638.x

[7] D. Benítez y H. Navarro, "Rendimiento de maíz-frijol en un tepetate en el segundo año de rehabilitación agrícola," Memorias del III Simposio Internacional sobre Suelos volcánicos endurecidos, pp. 279-286, 1996.

[8] M. Da Costa, "Etude exploratoire des modes de mise en valeur agroécologiques en terrains de cangahua habilités dans le nord de la sierra équatorienne, canton de Cayambe,” Memoire de fin de curs. Istom-CTI-UCE., p. 83, 2014.

[9] C. Zebrowski y A. Vicuña, "El cultivo de la canguahua en el medio campesino tradicional en el Ecuador," Memorias del III Simposio Internacional sobre Suelos volcánicos endurecidos, pp. 472-481, 1996.

[10] C. Castillo, "Evaluación de tres fuentes de materia orgánica (Champiñonaza, Bovinaza y Lombrihumus) en la habilitación de cangahua tipo C,” Tesis de grado: Universidad Central del Ecuador, Quito, 2015.

[11] Gobierno de Pichincha, "Plan de Desarrollo y Ordenamiento Territorial de la Parroquia de Alangasí 2012-2025,” 2012. [En línea] Available:

http://www.pichincha.gob.ec/phocadownload/leytransparencia/literal_ k/ppot/dmq/ppdot_alangasi.pdf.
[12] J. Moreno, J. Aguarón, F. Cano y M. Ecobar, "Validez, robustez y estabilidad en decisión multicriterio. Análisis de sensibilidad en el proceso analítico jerárquico,” Rev. R. Acad. Cien. Exact. Fis. Nat., vol. 92, nº 4, pp. 387-397, 1998.

[13] M. Ramírez, "El método de jerarquías analíticas de Saaty en la ponderación de variables. Aplicación al nivel de mortalidad y morbilidad en la provincia del Chaco," Comunicaciones Científicas y Tecnológicas de la Universidad Nacional del Nordeste, Corrientes, Argentina, 2004.

[14] J. Osorio y J. Orejuela, "El proceso de análisis jerárquico (AHP) y la toma de decisiones multicriterio. Ejemplo de aplicación,” Scientia Et Technica, vol. 14, n 39, pp. 247-252, 2008.

[15] Z. Lozano, "Muestreo con fines de caracterización y evaluación de propiedades de los suelos," Venesuelos, vol. 14, no 1, pp. 70-79, 2006.

[16] J. Valverde, J. Fernández, E. Jiménez, T. Vaca y F. Alarcón, "Microzonificación sísmica de los suelos del Distrito metropolitano de la ciudad de Quito,” 2001. [En línea]. Available: http://www.flacsoandes.edu.ec/libros/digital/51554.pdf. 